\title{
The Vicenza Wearable Artificial Kidney for Peritoneal Dialysis (ViWAK PD)
}

\author{
Claudio Ronco $^{\text {a }}$ Luciano Fecondini $^{\mathrm{b}}$

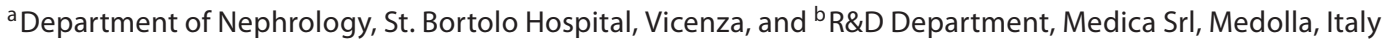

\section{Key Words}

Vicenza wearable artificial kidney for peritoneal dialysis • ViWAK PD • Wearable CAPD system

\begin{abstract}
Background: The study describes the structure and operational characteristics of a new wearable system for continuous ambulatory peritoneal dialysis (CAPD) for chronic kidney disease patients. Methods: We designed a wearable system consisting of: (1) a double lumen peritoneal catheter; (2) a dialysate outflow line; (3) a miniaturized rotary pump; (4) a circuit for dialysate regeneration featuring a waterproof container with 4 cartridges in parallel with a mixture of activated carbon and polystyrenic resins; (5) a filter for deaeration and microbiological safety; (6) a dialysate inflow line, and (7) a handheld computer as a remote control. The system has been tested circulating 12 liters of exhausted PD solution through the experimental adsorption unit at a rate of $20 \mathrm{ml} /$ min. Creatinine, $\beta_{2}$-microglobulin $\left(\beta_{2}-M G\right)$ and angiogenin were measured before and after the adsorption unit at baseline, and after 4 and $10 \mathrm{~h}$ of use. Results: The cartridges containing polystyrenic resin completely removed $\beta_{2}-M G$ and angiogenin from the fluid batch. Those with the activated carbon removed completely urea and creatinine. The final result was 11.2 liters of net solute clearance. The system is designed to be used as follows: The peritoneal cavity is loaded in the morning with 2 liters of fresh PD solution. After 2 h, when dialysate/plasma equilibration at approximately $50 \%$ has occurred, recirculation is activated for $10 \mathrm{~h}$ at a rate of 20 $\mathrm{ml} / \mathrm{min}$. After this period, recirculation stops and glucose is optionally added to the peritoneal cavity to achieve ultrafil-
\end{abstract}

tration if needed. After $2 \mathrm{~h}$ the fluid is drained and a 2-liter icodextrin exchange is performed overnight to achieve further ultrafiltration. The clearance provided by the minicycler is further increased by the 2-liter exchange and the overnight exchange. Therefore, the system operates $24 \mathrm{~h} /$ day and provides creatinine and $\beta_{2}-\mathrm{MG}$ clearance in the range of 15-16 liters/day, corresponding to a weekly clearance of 100-110 liters. The patient reduces the number of exchanges compared to CAPD and uses less fluid than in automated peritoneal dialysis (APD). Furthermore, the handheld computer allows for prescription and assessment of the therapy providing information on cartridge saturation, flow and pressure conditions and offering the possibility of remote wireless control of operations. Some problems still remain to be solved in the present configuration including the addition of an injection system for glucose and bicarbonate when needed, a system to reduce fibrin delivery to the sorbent and finally a more complex mixture of sorbents to make sure a complete removal of small molecules including urea is achieved. Conclusion: The wearable PD system may become a possible alternative to APD or CAPD reducing the time dedicated to perform exchanges and improving peritoneal dialysis adequacy and patient's rehabilitation.

Copyright $\odot 2007$ S. Karger AG, Basel

\section{Introduction}

The increased incidence of chronic kidney disease and the parallel increase of the prevalence of patients treated with renal replacement therapy are considered an important factor in the future allocation of national budgets for

\section{KARGER \\ Fax +4161306 1234 E-Mail karger@karger.ch} www.karger.com

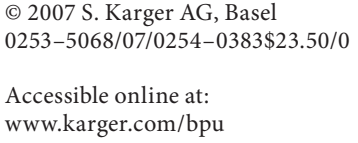

\footnotetext{
Dr. Claudio Ronco

Department of Nephrology, St. Bortolo Hospital

Viale Rodolfi 16

IT-36100 Vicenza (Italy)

Tel. +390444 753650, Fax +390444 753949, E-Mail cronco@goldnet.it
} 
healthcare. In this setting, the cost of personnel seems to overcome significantly the cost of technology and therefore, healthcare providers, either public or private, are trying to implement all possible measures to increase the portion of uremic patients treated at home.

Peritoneal dialysis (PD) is the most common form of home-based renal replacement therapy [1]. In spite of significant improvement in technique survival [2] and reduction of infectious complications [3], PD still remains underutilized in most countries [4]. Several barriers to the expansion of the PD program have been identified as possible causes of PD underutilization [5]. Among them, the commitment of patients and the time spent in performing the technique, together with the limitations imposed to the normal life, represent the most common factors pushing patients away from the choice of PD.

In this paper we present an innovative wearable system called Vicenza Wearable Artificial Kidney for Peritoneal Dialysis (ViWAK PD). The system is conceived to perform continuous ambulatory peritoneal dialysis (CAPD) requiring some maneuvers only in the morning and in the evening, and leaving the patient free during the day and at night. The concept of the ViWAK PD is based on the fact that a user-friendly interface and a miniaturized system reducing the number of maneuvers and liters of solution required to perform PD may contribute to a better acceptance by the patient and a wider application of this form of renal replacement therapy. The system is based on a combination of a long overnight dwell exchange and a continuous-flow PD during the day performed with a special catheter and a special minicycler utilizing a mixture of sorbents for regenerating the $\mathrm{PD}$ solution.

The paper describes the layout of the system and the results achieved in vitro with the ViWAK PD.

\section{Methods}

The project was divided into two parts: (A) the experimental phase to assess the capacity of the experimental adsorption unit to regenerate at least 12 liters of spent dialysate and remove three marker molecules of different molecular weight (creatinine 113 $\mathrm{Da}, \beta_{2}$-microglobulin $\left(\beta_{2}-\mathrm{MG}\right) 11,800 \mathrm{Da}$, and angiogenin 14,000 $\mathrm{Da})$, and (B) the design phase in which the system was miniaturized and conceived as a wearable device.

Phase A. We collected 6 bags of spent dialysate from 3 different CAPD patients and adjusted the volume to 12 liters discarding the excess. The dialysate was filtered through a small polysulfone filter (D-100; Medica Srl, Medolla, Italy) to remove fibrin and then it was circulated at a rate of $20 \mathrm{ml} / \mathrm{min}$ through an experimental adsorption unit made of 4 small cartridges in parallel and con- taining a mixture of activated charcoal and polystyrenic resin in proportion of 60 and $40 \%$ respectively. Activated charcoal mostly removes creatinine while polystyrenic resin mainly removes middle molecules. Data provided by previous literature were carefully analyzed to plan the experiments [6-10]. Samples before and after the adsorption unit were drawn at the beginning of the experiment and after 4 and $10 \mathrm{~h}$. Every experiment was done in triplicate.

Phase B. Based on the experimental results and the technical requirements concerning wearability and miniaturization, we asked a group of engineers to research and develop a new miniaturized system for continuous-flow PD with regeneration of spent dialysate (Medica Srl). The design of this minicycler had to meet specific priorities such as easy wearability, light weight and small size, safe connections of the circuitry to the catheter, user-friendly interface, to be battery-operated and to be capable of providing effective recirculation of PD solution at a rate of at least $20 \mathrm{ml} / \mathrm{min}$ for $>12 \mathrm{~h}$.

In order to enable a safe and effective PD fluid recirculation, the system had to be integrated with a double lumen catheter designed for continuous-flow PD. For this purpose we utilized the new dual lumen catheter called Ronco Cath (Medcomp, Harleysville, Pa., USA) [11]. The catheter is characterized by an inflow pathway ending with a fluid diffuser and an outflow pathway designed as a spiral classic Tenchkhoff catheter.

\section{Results}

Phase A. The results achieved in experimental phase $A$ are reported in figure 1. In particular, we observed an almost complete elimination of creatinine, $\beta_{2}-\mathrm{MG}$ and angiogenin from the fluid entering the adsorption unit with only a slight decrease in retention capacity after 10 $h$ of recirculation. The 'flow-through' value for creatinine, $\beta_{2}-\mathrm{MG}$ and angiogenin was $<10 \%$ after $10 \mathrm{~h}$ of use, confirming the capacity of the sorbent system to remove small and middle molecules. The final result in $10 \mathrm{~h}$ was a net clearance of 11.2 liters for creatinine and 11.4 and 11.1 liters for $\beta_{2}-\mathrm{MG}$ and angiogenin respectively. The study was intentionally limited to these three marker molecules for the moment since the final composition of the sorbent mixture is still experimental and not completely fixed. We are well aware that urease must be probably added to the mixture and the effluent will require a careful evaluation in terms of biocompatibility, acid base and osmotic force. For the moment however, the analysis of these marker molecules was designed to make the experiments as a 'proof of concept'.

Phase B. Based on the preliminary experimental results on the feasibility of dialysate regeneration, the ViWAK PD was conceived and developed according to the priorities announced for an easy-to-use wearable device. 


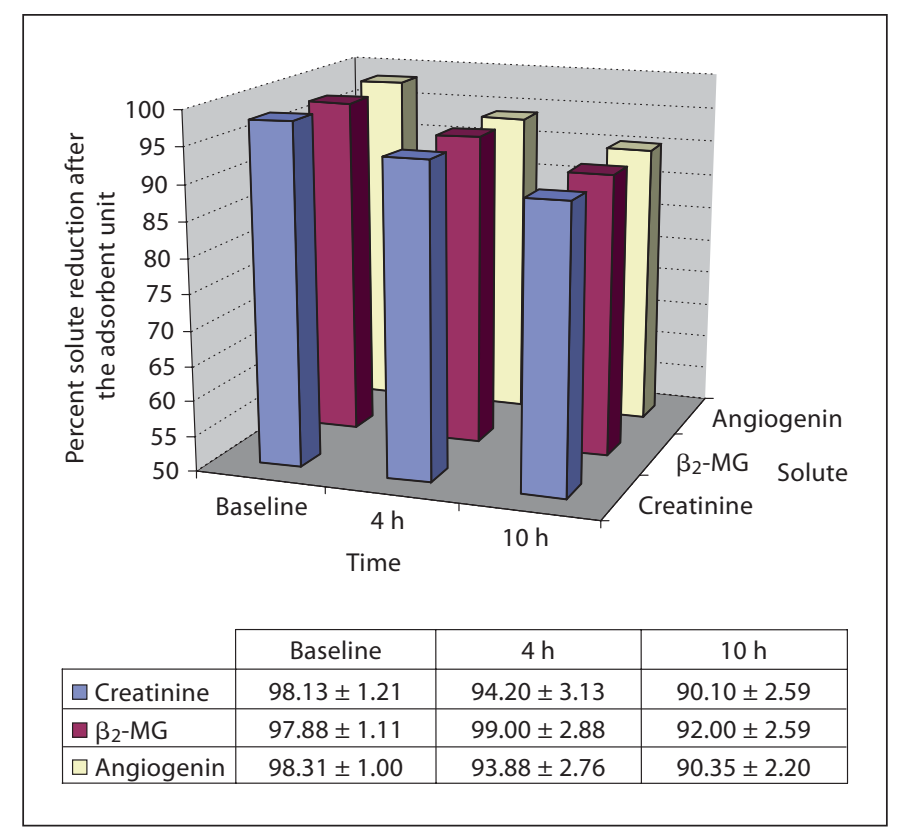

Fig. 1. Results obtained from the flow-through experiments carried out with spent dialysate circulated through the adsorbent unit. It can be noted that in all cases (experiments done in triplicate) the reduction ratio was still $>90 \%$ for the three marker molecules after $10 \mathrm{~h}$ of utilization of the sorbent.

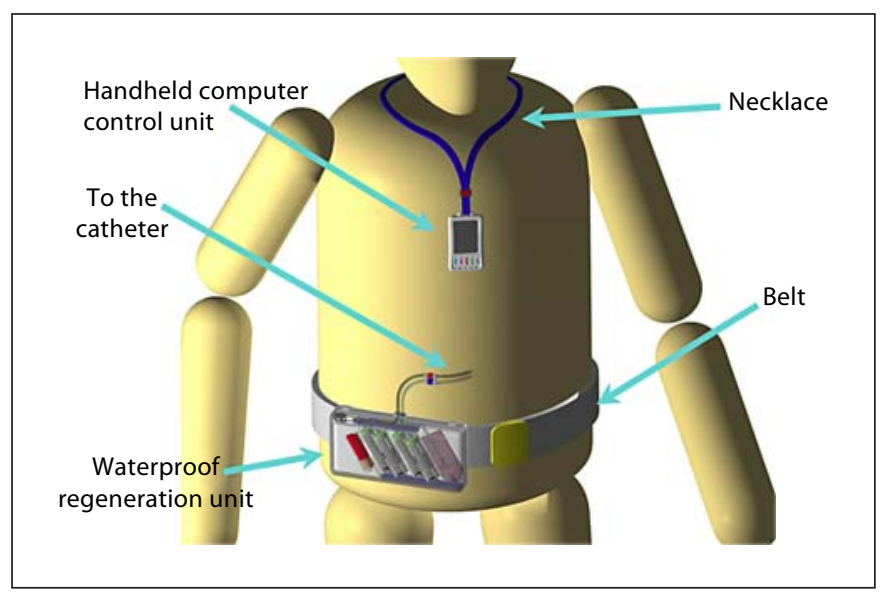

Fig. 2. Schematic representation of the ViWAK consisting of a belt with the waterproof adsorption unit, the connection to the catheter and the handheld computer for remote wireless control of operations.

A schematic view of the complete system is shown in figure 2. It is a daily, battery-operated adsorption system in which PD is recirculated and regenerated for $10 \mathrm{~h}$. The ViWAK PD utilizes a double lumen catheter (fig. 3) char-

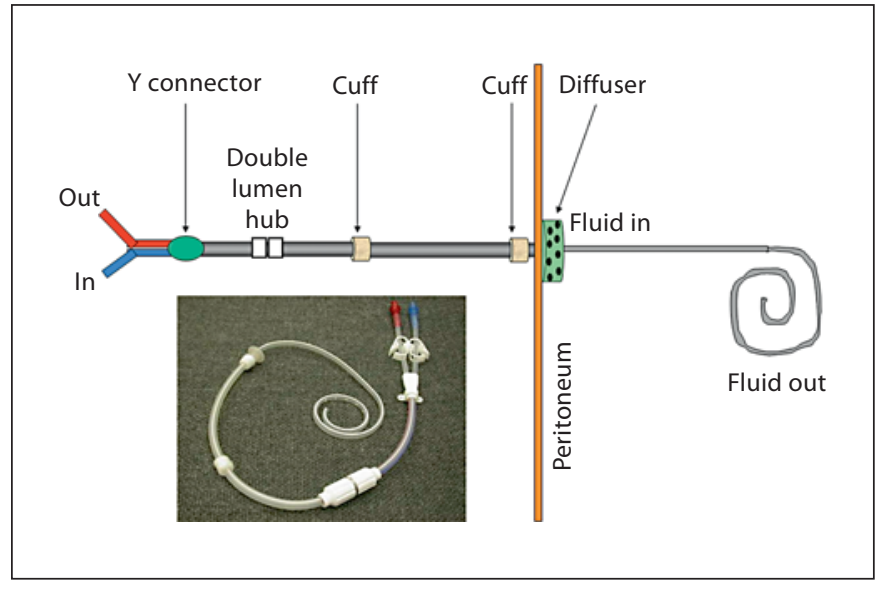

Fig. 3. The double lumen catheter used for the ViWAK. The inflow lumen ends with a diffuser while the outflow lumen begins with a spiral tube. This configuration ensures minimal intraperitoneal recirculation and maximal efficiency.

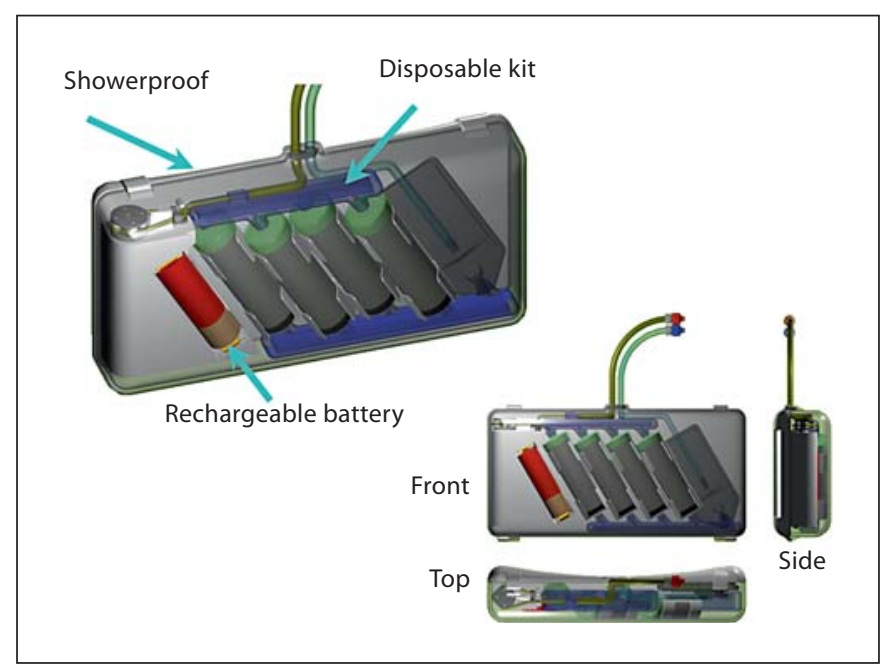

Fig. 4. Details of the waterproof container. The size of the device is $17 \times 8 \times 3 \mathrm{~cm}$ while total weight is approximately $200 \mathrm{~g}$.

acterized by a special configuration ensuring minimal intraperitoneal recirculation of the fluid regenerated in the extracorporeal adsorption unit. The details of the adsorption unit are shown in figures 4 and 5 . It consists of a waterproof device of small dimensions $(17 \times 8 \times 3 \mathrm{~cm})$ and a total weight of approximately $200 \mathrm{~g}$, containing a single-use kit designed to clean up to 12 liters of recirculated peritoneal solution. The kit is provided with circuitry, a sterile filter and debubbler, a safety pressure sensor, an adsorber cartridges pack and a rechargeable or single- 


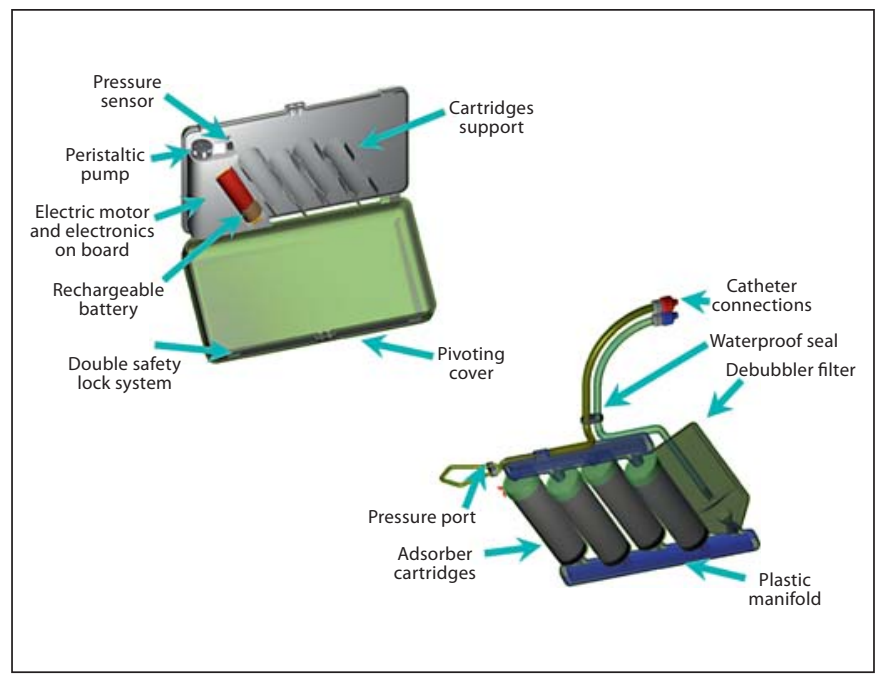

Fig. 5. Detail of the container once it is open and the single-use service pack. The container has embedded electronics and the rotary pump as well as the pressure sensor and the allocation for the service pack. The adsorption unit (service pack) features 4 sorbent cartridges placed in parallel and a $0.2-\mu \mathrm{m}$ filter for microbiological safety.

use battery. In the box are the electronic components and the small rotary pump with sensors. A dedicated handheld computer with a wireless connection to a PC and to the unit completes the equipment (fig. 6).

\section{Intended Use and Treatment Schedule}

The system is designed to be used as a simple mechanical aid to perform CAPD. A typical schedule could be summarized as follows: the peritoneal cavity is loaded in the morning with 2 liters of fresh PD solution. After approximately $2 \mathrm{~h}$, when $50 \%$ dialysate/plasma equilibration has occurred, recirculation is activated for $10 \mathrm{~h}$ at a rate of $20 \mathrm{ml} / \mathrm{min}$ (range between 15 and 30). After this period, recirculation stops and a complete equilibration is obtained in the remaining indwelling solution. Glucose could be optionally added to the peritoneal cavity to achieve ultrafiltration if needed through a line connected to a small reservoir placed on the belt. This function can be activated by the patient via handheld computer. After $2 \mathrm{~h}$ the fluid is drained and a 2-liter icodextrin exchange is performed overnight to achieve further ultrafiltration. Assuming a daily clearance of 12 liters obtained by the minicycler, as demonstrated by the experimental findings, an additional clearance of $4-5$ liters can therefore be provided by the equilibrated solution drained in the eve-

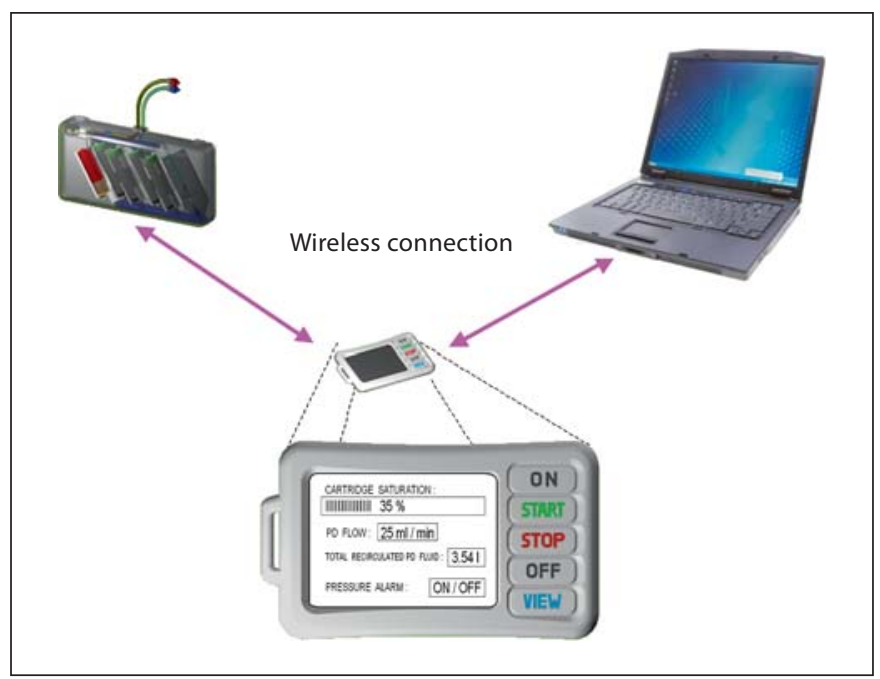

Fig. 6. The system operates via a handheld computer with remote wireless connection to a PC and to the device. The user-friendly display of the computer provides information on cartridge saturation and allows for easy operations controlled by the patient.

ning and by the overnight exchange. Such efficiency guarantees a weekly creatinine clearance $>100$ l/week and may represent an important treatment for larger patients unable to meet the adequacy targets proposed by the international guidelines [12].

\section{Discussion}

Attempts to improve the efficiency of CAPD and automated peritoneal dialysis (APD) have led to continuous-flow PD [13]. One of the major limitations imposed by continuous-flow PD is the requirement of large amounts of fluid to allow for a significant increment of clearance. An alternative is offered by a system to regenerate small amounts of dialysis solution recirculated in the peritoneal cavity. Sorbents in line with improved dialysis catheters have the potential to reduce the cost of dialysis fluid by fluid regeneration, and to remove toxic products, and they offer the major prospect of increasing the efficiency of PD.

Possible sorbents for such a function are activated carbons, ion-exchange resins or non-ionic macroporous resins. Adsorptive capacity for all sorbents is enhanced by inducing a high surface porosity and large surface area. Non-polar solutes are better adsorbed from aqueous solution than are polar solutes. With traditional uncoated 
carbon, small toxins (creatinine, uric acid, hippuran, and vitamin $\mathrm{B}_{12}$ ) are removed. Theoretically, modern carbon sorbents are capable of removing middle molecular weight toxins although this function seems better accomplished by synthetic hydrophobic resins.

It is possible today to create resin beads with a large adsorptive surface area. Such a resin may have a different pore size distribution capable of removing molecules from 8-30 kDa, such as $\beta_{2}$-MG, leptin, IL-6, IL-18, angiogenin and others. The crucial aspect regards the packing density of the sorbent: this aspect in fact characterizes the functionality of the cartridge and the resistance to flow. In the case of peritoneal dialysate, the viscosity is for sure less than that of blood; nevertheless, we should not neglect the significant proteinaceous component and the accumulation of fibrin in the spent peritoneal dialysate. For this reason a minifilter placed in series with the sorbent may contribute to an improved functionality and survival of the sorbent bed. Hydrophobic solutes are removed by carbon and non-ionic macroporous resins, while adsorption through chemical affinity requires ionexchange resins and chemisorbents. For this reason, a combination of sorbents may represent the most suitable way to provide an efficient and safe dialysate regeneration. A combination of zirconium, charcoal and urease has been used to regenerate dialysate [7-10].

In our system, the mixture of adsorbents was placed in a parallel cartridge pack to improve adsorption and reduce resistance to flow. Continuous-flow PD can therefore be performed recirculating the 2 liters of indwelling solution for a prolonged period of time.

Sorbents offer singularly unique attributes for enhancing PD as they do in hemodialysis. In addition, they can be designed to remove toxic glucose degradation products and aldehydes from heat-sterilized dialysis fluid. Sorbents have been investigated for their role in enhancing solute removal [14-17]. This has been applied to PD in order to achieve remarkable increments in urea and creatinine clearances [14-15]. Sorbents offer the theoretical advantage of removing also protein-bound metabolic toxins $[16,17]$.

The evolution of technology in recent years has made it possible to reduce the size and weight of pieces of equipment that in the past would have occupied the space of an entire room. This has been the case with computers moving from mainframes to personal PCs to handheld computers. In some cases, especially in the medical device area, the miniaturization has reached the point where devices can be worn by the patient or even be implanted. This has been the case with pacemakers. In the field of dialysis, miniaturization occurred in the early 1980s when large flat dialyzers were substituted by small compact hollow-fiber dialyzers. However, the size of dialysis machines has remained unchanged for years and today the size is approximately the same as the first dialysis machines were.

Recently, Gura et al. $[18,19]$ have proposed to modify the design of the extracorporeal dialysis system not only miniaturizing it, but also making it wearable by the patient. Other attempts to make intracorporeal continuous blood purification were done in the past thanks to the design of special 'hemofiltration' catheters $[20,21]$ or continuous flow-through PD [22]. In this paper we have described another approach which is based on the concept of continuous-flow PD. The system is based on the combination of a daily continuous-flow PD actuated by a wearable minicycler and a small dialysate regeneration system, and the addition of one overnight exchange done with icodextrin-containing PD solution. According to our preliminary data, the system could become an alternative to CAPD or APD providing improved efficiency ( $>100$ liters of clearance per week) and increased freedom for the patient. Many problems remain to be solved but the concept is proven and we hope that this approach will spur a new interest towards miniaturization of dialysis devices for an improved benefit of our patients.

\section{Acknowledgement}

We would like to thank Mr. Luca Pignatti for his important contribution to the project.

\section{Disclosure}

The ViWAK PD system has not been patented on purpose to allow investigators of the world to continue and enhance research in this field without limitations or restrictions.

References

1 Finkelstein FO, Finkelstein SH, Troidle LK: The role of peritoneal dialysis in the management of the patient with chronic kidney disease. Contrib Nephrol. Basel, Karger, 2006, vol 150, pp 235-239.

2 Blake PG, Finkelstein FO: Why is the proportion of patients doing peritoneal dialysis declining in North America? Perit Dial Int 2001;21:107-114.

-3 Kiernan L, Kliger A, Gorban-Brennan N, Juergensen P, Tesin D, Vonesh E, Finkelstein F: Comparison of continuous ambulatory peritoneal dialysis-related infections with different 'Y-tubing' exchange systems. J Am Soc Nephrol 1995;5:1835-1838. 
4 Ronco C, Dell'Aquila R, Rodighiero MP, Di Loreto $\mathrm{P}$, Spanò E: Integration of peritoneal dialysis in the treatment of uremia. Contrib Nephrol. Basel, Karger, 2006, vol 150, pp 226-234.

5 Troidle L, Kliger A, Finkelstein F: Barriers to utilization of chronic peritoneal dialysis in network \#1, New England. Perit Dial Int 2006;26:452-457

6 Shapiro W: Sorbent dialysis; in Nissenson A, Fine R (eds): Clinical Dialysis, ed 4. New York, McGraw-Hill, 2005, pp 981-986.

7 Ash S: The Allient Dialysis System. Semin Dialysis 2004; 17:164-166.

8 Shapiro W: REDY sorbent hemodialysis system; in Nissenson AR, Fine RN (eds): Dialysis Therapy, ed 3. Philadelphia, Hanley \& Belfus, 2002, pp 215-219.

-9 Roberts M: The regenerative dialysis (REDY) sorbent system. Nephrology 1998;4:275278.

10 Tetta C, Bellomo R, Brendolan A, Piccinni P, Digito A, Dan M, Irone M, Lonnemann G, Moscato D, Buades J, La Greca G, Ronco C: Use of adsorptive mechanisms in continuous renal replacement therapies in the critically ill. Kidney Int 1999;56(suppl 72):S15-S19.
1 Ronco C, Dell'aquila R, Rodighiero MP, Di Loreto P, Nalesso F, Spano E, Parkhill R, Amerling R, Levin N: The 'Ronco' catheter for continuous-flow peritoneal dialysis. Int J Artif Organs 2006;29:101-112.

12 Bargman J, Thorpe K, Churchill D: Relative contribution of residual renal function and peritoneal clearance to adequacy of dialysis: a re-analysis of the CANUSA study. J Am Soc Nephrol 2001;12:2158-2162.

13 Ronco C, Amerling R: Continuous-flow peritoneal dialysis: current state-of-the-art and obstacles to further development. Contrib Nephrol. Basel, Karger, 2006, vol 150, pp 310-320.

14 Amerling R, DeSimone L, Inciong-Reyes R, Pangilinan A, Folden T, Ronco C, Gotch FA, Levin N: Clinical experience with continuous-flow and flow-through peritoneal dialysis. Semin Dial 2001;14:388-390.

15 Amerling R, Glezerman I, Savransky E, Dubrow A, Ronco C: Continuous-flow peritoneal dialysis: current perspectives. Contrib Nephrol. Basel, Karger, 2003, vol 140, pp 294-304.

16 Diaz-Buxo JA: Streaming, mixing, and recirculation: role of the peritoneal access in continuous-flow peritoneal dialysis (clinica considerations). Adv Perit Dial 2002;18:8790
17 Winchester JF, Amerling R, Harbord N, Capponi V, Ronco C: The potential application of sorbents in peritoneal dialysis. Contrib Nephrol. Basel, Karger, 2006, vol 150, pp 336-343.

18 Gura V, Beizai M, Ezon C, Rambod E: Continuous renal replacement therapy for congestive heart failure: the wearable continuous ultrafiltration system. ASAIO J 2006;52: 59-61.

19 Gura V, Beizai M, Ezon C, Polaschegg HD: Continuous renal replacement therapy for end-stage renal disease. The wearable artificial kidney. Contrib Nephrol. Basel, Karger, 2005, vol 149, pp 325-333.

20 Handley HH, Gorsuch R, Peters H, Cooper TG, Bien RH, Levin NW, Ronco C: Slow continuous intravenous plasmapheresis: clinical applications and hemostability of extracorporeal ultrafiltration. Contrib Nephrol. Basel, Karger, 2005, vol 149, pp 334-342.

21 Handley HH, Gorsuch R, Levin NW, Ronco C: Slow continuous intracorporeal plasmapheresis for acute fluid overload. Blood $\mathrm{Pu}-$ rif 2003;21:72-78.

22 Roberts M, Ash SR, Lee DB: Innovative peritoneal dialysis: flow-thru and dialysate regeneration. ASAIO J 1999;45:372-378. 